\title{
Prognostic value of CD4+ lymphocytes in pleural cavity of patients with non-small cell lung cancer
}

\author{
K Takahashi, S Saito, Y Kamamura, M Katakawa, Y Monden
}

\begin{abstract}
Background-For patients with nonsmall cell lung cancer the TNM staging system and other conventional prognostic factors fail to predict accurately the outcome of treatment and survival. This study attempts to determine the prognostic value for survival of the proportions of CD4+ lymphocytes in the pleural cavity (PLY) of patients with resectable nonsmall cell lung cancer.

Methods-Lymphocytes in the pleural cavity separated from 51 patients with non-small cell lung cancer were examined by flow cytometry to measure the proportions of CD4+ PLY. Univariate and multivariate analyses were performed to assess the association between the proportion of CD4+ PLY and survival.

Results-The 5 year survival rate of patients with percentage CD4+ PLY of $\leqslant 30 \%$ was $84 \%$ whereas that of patients with \%CD4+ PLY $>30 \%$ was $26.9 \%$. The difference in survival between the \%CD4+ PLY $\leqslant 30 \%$ and \%CD4+ PLY $>30 \%$ groups was significant $(p<0.0001)$. The \%CD4+ PLY in those who survived for 5 years was significantly lower than that in the patients who died within 5 years $(p<0.0001)$. The difference in survival between patients with stage IA and IB lung cancer with \%CD4+ PLY $\leqslant 30 \%$ and those with $\%$ CD4+ PLY $>30 \%$ was also significant (p $=0.015)$. Multivariate analysis showed that the proportion of CD4+ PLY (hazard ratio $=6.9,95 \% \mathrm{CI} 0.045$ to 0.47 ) and nodal status (hazard ratio $=22.7,95 \%$ CI 0.006 to 1.806) are significant and independent prognostic factors for the survival of patients with lung cancer.

Conclusions-The proportion of CD4+ PLY may help to select patients who are likely to have a poorer prognosis after surgery and therefore may be suitable for consideration of adjuvant treatments. These results need confirmation in a larger prospective study.

(Thorax 2001;56:639-642)
\end{abstract}

Keywords: lung cancer; prognosis; CD4

For patients with lung cancer, conventional prognostic factors such as TNM stage, the extent of lymph node metastasis, and performance status currently determine the choice of treatment. However, considerable differences in outcome after treatment are seen between patients with the same initial stage and performance status. ${ }^{12}$ The influence of additional variables may explain this heterogeneous prognosis. New prognostic factors are needed to improve the understanding of the biological behaviour of lung cancer and to recognise patients with a good or poor prognosis.

The cell mediated arm of immune surveillance plays a major role in the destruction of cancer cells. A quantitative measure of cell mediated immunity is the measurement of proportions of CD4+ and CD8+ lymphocytes. The proportion of CD4+ cells in peripheral blood lymphocytes (PBL) in patients with malignancy decreases according to tumour progression and the decrease in the proportion of $\mathrm{CD} 4+\mathrm{PBL}$ is associated with a poor prognosis..$^{3-5}$ Impaired cellular immunity can be associated with a change in the proportions of these cells.

Malignant pleuritis is frequently observed in lung cancer ${ }^{6-11}$ and patients with malignant pleuritis have a poor prognosis. In malignant pleural effusions CD4+ cells are dominant, and the proportion of CD8+ cells is significantly lower than that of CD4+ cells. ${ }^{12-15}$ In contrast, the proportion of CD4+ cells in the pleural cavity of lung cancer patients without malignant effusion is significantly lower than that of CD8+ cells. ${ }^{16}{ }^{17}$ Invasion of cancer cells into the pleural cavity may be affected by both the nature of the cancer cells and host factors of patients with lung cancer. ${ }^{18}$

We have examined the proportions of CD4+ and CD8+ lymphocytes in the pleural cavity (PLY) of patients with resectable non-small cell lung cancer to determine whether the proportions of these cells have a prognostic value for patient survival.

\section{Methods}

PATIENTS

The study subjects comprised 51 patients (42 men) of median age 66 years (range 46-83) with resectable primary lung cancer not associated with malignant pleural effusion. None of the patients had received any anticancer therapy prior to the study. Computed tomographic scanning and magnetic resonance imaging were used to determine whether a pleural effusion was present. The clinical characteristics of the patients are summarised in table 1. Histologically, there were 25 squamous cell carcinomas, 24 adenocarcinomas, and two large cell carcinomas. The TNM classification system (1997 international staging system for non-small cell lung cancer) was used for staging the disease. The degree of pleural invasion by lung cancer $(p$ factor) was grade 0 (no visceral pleural invasion) in 34 patients, grade 1 (pleural invasion limited 
Table 1 Clinical features of study patients

\begin{tabular}{ll}
\hline Features & No \\
\hline No of patients & 51 \\
M:F & $42: 9$ \\
Age (years) & \\
$\quad$ Median & 66 \\
$\quad$ Range & $46-83$ \\
Histology & \\
Squamous cell & 25 \\
Adenocarcinoma & 24 \\
Large cell & 2 \\
Clinical stage & \\
IA & 23 \\
IB & 4 \\
IIA & 4 \\
IIB & 5 \\
IIIA & 8 \\
IIIB & 7 \\
\hline
\end{tabular}

to the visceral pleura) in six patients, grade 2 (pleural invasion extending beyond the visceral pleura but not extending to the neighbouring lobe or chest wall) in three patients, and grade 3 (pleural invasion extending beyond the neighbouring lobe or chest wall) in eight patients. The degree of pleural invasion by cancer was
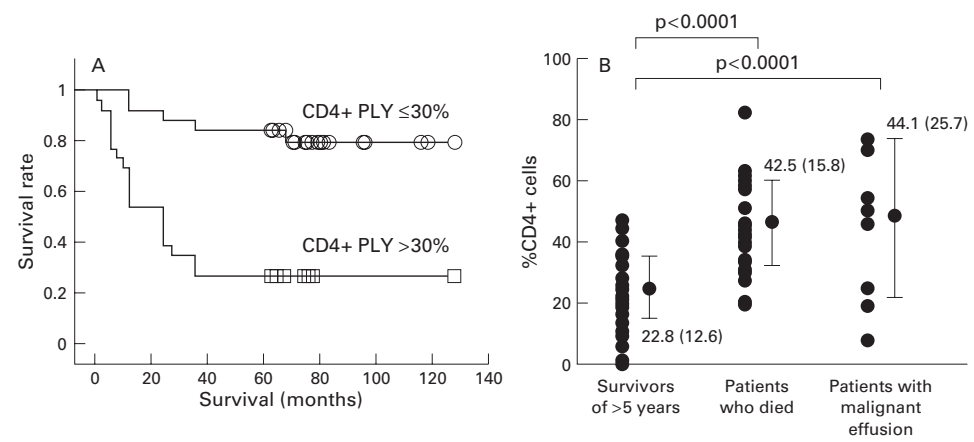

Figure 1 (A) Log rank survival of patients with $\% C D 4+P L Y \leqslant 30 \%$ ( $n=24)$ or $>30 \%$ $(n=27)$. (B) There were eight patients with malignant pleural effusions who were not candidates for surgery.
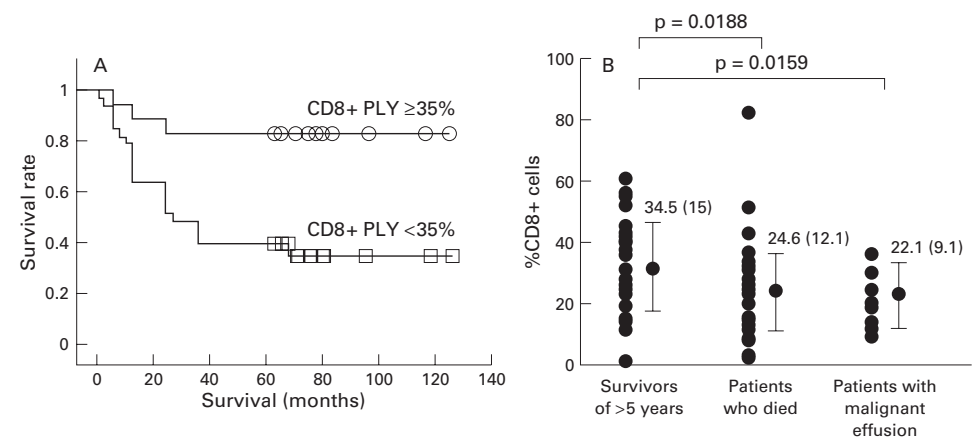

Figure 2 (A) Log rank survival of patients with $\% C D 8+P L Y \geqslant 35 \%$ ( $n=18$ ) or $<35 \%$ $(n=33)$. (B) There were eight patients with malignant pleural effusions who were not candidates for surgery.
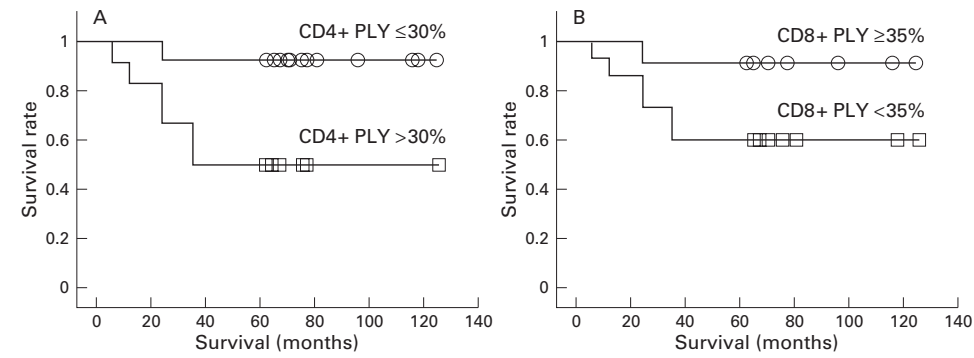

Figure 3 Log rank survival of patients with $(A) \% C D 4+P L Y \leqslant 30 \%(n=14)$ or $>30 \%$ $(n=12)$ and $(B) \% C D 8+P L Y \geqslant 35 \%(n=11)$ or $<35 \%(n=15)$ in 26 patients with stage IA and IB lung cancer. determined by histological examination; 29 patients were classified as n0, seven as $n 1,13$ as $\mathrm{n} 2$, and two as $\mathrm{n} 3$. All of these patients were confirmed to have no malignant pleural effusion at the time of thoracotomy. Eight other lung cancer patients with malignant pleural effusion, who were not candidates for surgery, were also examined.

ISOLATION OF LYMPHOCYTES IN PLEURAL CAVITY After obtaining informed consent, pleural lavage was performed as previously described. ${ }^{16}{ }^{17}$ At thoracotomy it was confirmed that no malignant pleural effusion was present. The pleural cavity was then lavaged with $1000 \mathrm{ml}$ of $0.9 \% \mathrm{NaCl}$ solution at $37^{\circ} \mathrm{C}$. The lavaged fluid was collected aseptically in heparinised $(10 \mathrm{U} / \mathrm{ml})$ centrifuge bottles and centrifuged at $1200 \mathrm{rpm}(400 \mathrm{~g})$ for 10 minutes. The cell pellets were resuspended in $15 \mathrm{ml}$ phosphate buffered saline and mononuclear cells were separated from the lavage fluid by discontinuous gradient centrifugation in lymphocyte separation medium. The absence or presence of malignant cells among the washed mononuclear cells was determined morphologically using May-Grunwald Giemsa staining. The lavage and isolation methods used yielded a mean $22.3\left(\right.$ SD26.9) $\times 10^{6}$ (range $1-117 \times 10^{6}$ ) mononuclear cells per patient. These cells consisted of 56.7 (13.1)\% PLY. Isolation of PLY was immediately followed by FACScan analysis.

FLOW CYTOMETRY

A direct immunofluorescence assay was used to detect the surface markers of PLY. Monoclonal antibodies (OKT4a and OKT8) conjugated with fluorescein isothiocyanate (Ortho Diagnostics, Raritan, NJ, USA) were added to cell suspensions and the mixtures were incubated for 30 minutes at $4^{\circ} \mathrm{C}$. The cells were then washed, resuspended in $0.1 \mathrm{ml}$ phosphate buffered saline, and analysed with a FACScan flow cytometer. ${ }^{16} 17$ The monoclonal antibodies used were directed against lymphocyte antigens of CD4 and CD8. Before FACScan analysis, dead cells and non-lymphoid cells were excluded from analysis by light scatter gating.

\section{STATISTICAL ANALYSIS}

The statistical differences were examined by the Mann-Whitney U test. A p value of $<0.05$ was considered to be significant. Survival curves were calculated according to the method of Kaplan and Meier. ${ }^{19}$ The groups were compared by log rank test. ${ }^{20}$ Multivariate relationships were analysed according to Cox's proportional hazards linear model and the SAS program package. ${ }^{21}$

\section{Results}

The median \%CD4+ PLY was 30.3\% (5-95th percentile 9.5-60; range 6.4-82.7). Accordingly, the cutoff value for survival analysis in this study was assessed by $30 \%$ of CD $4+$ PLY. The 5 year survival rate of lung cancer patients with $\%$ CD $4+$ PLY $\leqslant 30 \%$ was $84 \%$ while that of patients with $\%$ CD $4+$ PLY $>30 \%$ was $26.9 \%$. The median survival time of patients with 
Table 2 Univariate survival analysis of patients with lung cancer

\begin{tabular}{|c|c|c|c|}
\hline Variable & Hazard ratio & $95 \% C I$ & $p$ Value \\
\hline Proportion of CD4+ PLY & & & 0.0004 \\
\hline$\leqslant 30 \%$ & 0.168 & 0.062 to 0.454 & \\
\hline$>30 \%$ & 1.000 & & \\
\hline Proportion of CD8+ PLY & & & 0.0089 \\
\hline$\geqslant 35 \%$ & 0.198 & 0.059 to 0.677 & \\
\hline$<35 \%$ & 1.000 & & \\
\hline $\mathrm{p}$ factor & & & 0.0015 \\
\hline 0 & 0.173 & 0.063 to 0.47 & \\
\hline 1 & 0.566 & 0.178 to 1.801 & \\
\hline 2 & 1.297 & 0.332 to 5.068 & \\
\hline 3 & 1.000 & & \\
\hline Nodal status & & & 0.0036 \\
\hline 0 & 0.92 & 0.18 to 0.464 & \\
\hline 1 & 0.232 & 0.4 to 1.351 & \\
\hline 2 & 0.418 & 0.088 to 1.981 & \\
\hline 3 & 1.000 & & \\
\hline Clinical stage & & & 0.0037 \\
\hline IA & 0.076 & 0.021 to 0.266 & \\
\hline IB & 0.335 & 0.085 to 1.319 & \\
\hline IIA & 0.125 & 0.015 to 1.042 & \\
\hline IIB & 0.459 & 0.132 to 1.594 & \\
\hline IIIA & 0.408 & 0.128 to 1.296 & \\
\hline IIIB & 1.000 & & \\
\hline Histology & & & 0.859 \\
\hline Squamous cell & 0.813 & 0.104 to 6.359 & \\
\hline Adenocarcinoma & 1.023 & 0.133 to 7.836 & \\
\hline Large cell & 1.000 & & \\
\hline
\end{tabular}

PLY $=$ pleural cavity.

\%CD4+ PLY $>30 \%$ was 24 months. The difference in survival between the \%CD4+ PLY $\leqslant 30 \%$ group and the $\%$ CD $4+$ PLY $>30 \%$ group was significant $(\mathrm{p}<0.0001$; fig $1 \mathrm{~A})$. The $\%$ CD $4+$ PLY in those who survived 5 years was significantly lower than that in the patients who died in less than 5 years $(\mathrm{p}<0.0001$; fig $1 \mathrm{~B})$. The $\% \mathrm{CD} 4+\mathrm{PLY}$ in malignant effusions of lung cancer patients who were not candidates for surgery was significantly higher than in those who survived for 5 years $(\mathrm{p}<0.0001$; fig $1 \mathrm{~B})$.

The median \%CD8+ PLY was $33.2 \%$ (5-95th percentile 9.2-53.1; range 3.8-61.7). Accordingly, the cutoff value for survival analysis in this study was assessed by $35 \%$ of CD8+ PLY. The 5 year survival rate of lung cancer patients with \%CD8+ PLY $\geqslant 35 \%$ was $83.3 \%$ and that of patients with \%CD8+ PLY $<35 \%$ was $36.4 \%$. The median survival time of patients whose $\%$ CD $8+$ PLY was $<35 \%$ was 27 months. The difference in survival between the $\% \mathrm{CD} 8+$

Table 3 Multivariate survival analysis of patients with lung cancer based on Cox's hazard regression model

\begin{tabular}{llll}
\hline Variable & Hazard ratio & $95 \%$ CI & p Value \\
\hline $\begin{array}{l}\text { Proportion of CD4+ PLY } \\
\quad 30 \%\end{array}$ & 0.145 & 0.045 to 0.47 & 0.0013 \\
$\quad \begin{array}{l}\text { >30\% } \\
\text { Proportion of CD8+ PLY }\end{array}$ & 1.000 & & \\
$\quad$ & & \\
$\quad 35 \%$ & 0.294 & 0.073 to 1.179 & \\
$\quad<35 \%$ & 1.000 & & 0.1097 \\
p factor & & & \\
0 & 4.223 & 0.205 to 87.022 & \\
1 & 6.166 & 0.311 to 122.389 & \\
2 & 31.094 & 1.193 to 810.683 & \\
3 & 1.000 & & \\
Nodal status & & & \\
0 & 0.044 & 0.006 to 0.339 & \\
1 & 0.164 & 0.015 to 1.806 & \\
2 & 0.199 & 0.035 to 1.13 & \\
3 & 1.000 & & \\
Clinical stage & & & \\
IA & 0.025 & 0.001 to 0.597 & \\
IB & 0.018 & 0.001 to 0.481 & \\
IIA & 0.133 & 0.004 to 4.685 & \\
IIB & 0.114 & 0.006 to 2.2 & \\
IIIA & 0.102 & & \\
IIIB & 1.000 & & \\
\hline
\end{tabular}

PLY = pleural cavity.
PLY $\geqslant 35 \%$ group and the $\%$ CD $8+$ PLY $<35 \%$ group was significant $(\mathrm{p}=0.0026$; fig $2 \mathrm{~A})$. The $\%$ CD8+ PLY in those who survived 5 years was significantly higher than that in the patients who died within 5 years $(p=0.0188$; fig $2 B)$. The $\%$ CD8+ PLY in malignant effusions of lung cancer patients was significantly lower than that in 5 year survivors ( $\mathrm{p}=0.0159$; fig $2 \mathrm{~B}$ ). The proportions of PLY expressing CD3, CD16, CD25, CD57, or HLA-DR were not associated with the prognosis of the patients with lung cancer (data not shown).

The 5 year survival rate of patients with stage IA and IB lung cancer with \%CD4+ PLY $\leqslant 30 \%$ was $92.9 \%$ and that of patients with stage IA and IB disease with \%CD4+ PLY $>30 \%$ was $50 \%$. The difference in survival between the $\%$ CD $4+$ PLY $\leqslant 30 \%$ group and the $\%$ CD $4+$ PLY $>30 \%$ group was significant $(\mathrm{p}=0.015$; fig $3 \mathrm{~A})$. The 5 year survival rate of patients with stage IA and IB lung cancer with $\%$ CD8+ PLY $\geqslant 35 \%$ was $90.9 \%$ and that of patients with stage IA and IB cancer with $\%$ CD $8+$ PLY $<35 \%$ was $60 \%$. The difference in survival between the \%CD8+ PLY $\geqslant 35 \%$ group and the $\%$ CD $8+$ PLY $<35 \%$ group was not significant in these patients (fig $3 \mathrm{~B}$ ).

An examination of other factors that correlated with prognosis is shown in table 2. In addition to proportions of CD4+ PLY and CD8+ PLY, p factor, nodal status, and clinical stage were significantly associated with the prognosis.

Multivariate analysis (Cox proportional hazard models) clearly demonstrated that the proportion of CD4+ PLY and nodal status were the most important prognostic factors (table 3 ), and they were independently associated with the prognosis of the patients with lung cancer.

\section{Discussion}

Lung cancer patients with \%CD4+ PLY $\leqslant 30 \%$ are associated with a favourable prognosis. Multivariate analysis shows that the proportion of CD4+ PLY and nodal status are significant and independent prognostic factors for the survival of patients with lung cancer. Moreover, the proportion of CD4+ PLY can serve as a prognostic factor for patients with stage IA and IB non-small cell lung cancer.

The proportion of CD4+ cells in PBL in patients with malignancy decreases according to tumour progression and the decrease in the proportion of CD4+ PBL is associated with a poor prognosis. ${ }^{3-5}$ In this study, however, we found no significant change in the proportion of CD4+ PBL or CD8+ PBL in lung cancer patients according to tumour progression and the proportion of $\mathrm{CD} 4+\mathrm{PBL}$ or $\mathrm{CD} 8+\mathrm{PBL}$ was not associated with the prognosis of the patients with lung cancer (data not shown). Our subjects were patients with resectable non-small cell lung cancer. The proportion of CD4+ PBL or CD8+ PBL might be associated with the prognosis of the lung cancer patients with distant metastasis. Invasion of lung cancer cells into the pleural cavity may affect the nature of the PLY and distant metastasis of lung cancer cells may affect the nature of PBL. 
The proportions of CD4+ and CD8+ tumour infiltrating lymphocytes (TIL) are similar to those in PBL of patients with malignancy. ${ }^{22-25}$ In lung cancer patients, however, the proportion of CD8+ TIL is significantly higher than that of CD8+ PBL. ${ }^{26}{ }^{27}$ The proportion of CD4+ TIL increases and that of CD8+ TIL decreases according to tumour progression in patients with malignancy. ${ }^{22-27}$ The increase in the proportion of CD4+ TIL and the decrease in the proportion of CD8+ TIL are associated with a poor prognosis. ${ }^{22-27}$ In malignant peritoneal fluid the proportion of CD8+ lymphocytes decreases with progression of cancer cells. ${ }^{28}$ Following the changes in the population of lymphocytes in the peritoneal fluid of cancer patients may be useful for determining the prognosis. ${ }^{29}$

CD4+ lymphocytes can directly lyse tumour cells in an HLA class II restricted fashion. ${ }^{30} 31$ Most tumour specific CD4+ TIL and CD4+ lymphocytes in malignant peritoneal fluid may recognise tumour antigens presented on HLA class II molecules. CD4+ TIL and CD4+ lymphocytes in malignant peritoneal fluid of cancer patients may therefore play an important role in tumour regression mediated by the immune system. In malignant pleural effusions CD4+ PLY are dominant. ${ }^{12-15}$ By contrast, the proportion of CD4+ PLY in lung cancer patients without malignant pleural effusions is significantly lower than that of CD8+ PLY. ${ }^{16} 17$ In the pleural cavity changes in the population of lymphocytes may also occur according to tumour progression, as occurs in the tumour and the peritoneal cavity. The majority of tumour specific CD4+ PLY may also recognise tumour antigens presented on HLA class II molecules. These facts suggest that CD4+ PLY of lung cancer patients may play an important role in tumour regression, as do TIL and lymphocytes in malignant peritoneal fluid. The key to inducing the most potent killing response to a tumour is therefore the activation of CD4+ lymphocytes.

CD4+ PLY may be used diagnostically to select patients who are likely to have a poorer prognosis after surgery. How cancer cells invade the pleural cavity is still unknown. We believe that the most likely mechanism is by spread through lymphatic vessels. Cytological examination of pleural effusions or pleural lavage fluid would seem to be an important method for detecting invasion of lung cancer cells, but recognition of lung cancer cells by PLY might be more sensitive than cytological examination.

In conclusion, our retrospective study indicates that estimation of the proportion of CD4+ PLY is useful as a clinical biomarker which is related to the outcome of patients with resectable non-small cell lung cancer. If the current findings can be confirmed in large prospective studies, then measurement of the proportion of CD4+ PLY may become a useful clinical tool to select patients who are likely to have a poorer prognosis after surgery and who may be suitable for consideration of adjuvant treatments.
1 Mountain C. A new international staging system for lung cancer. Chest 1986;89:225-33S.

2 Shields $T$. The significance of ipsilateral mediastinal lymph node metastasis (N2 disease) in non-small cell carcinoma of the lung: a commentary. Ann Thorac Surg 1990;48:4853.

3 Gemignani M, Maiman M, Fruchter RG, et al. CD4 ymphocytes in women invasive and preinvasive cervical neoplasia. Gynecol Oncol 1995;59:364-9.

4 Greenstein A, Pecht M, Kaver I, et al. Characterization of peripheral blood T-cell subpopulation of bladder cancer patients. Urol Res 1991;19:219-22.

5 Kaver I, Pecht M, Trainin N, et al. T lymphocyte subset and function in the peripheral blood of patients with urological cancer. Oncology 1992;49:108-13.

6 Chretien J. Quelques aspects immunologiques des maladies de la pleure. Presse Med 1970;78:921-5.

7 Pattersson T, Klockars M, Hellstorm PE, et al. T and B lymphocytes in pleural effusions. Chest 1978;73:49-51.

8 Hausher F, Yabro JW. Diagnosis and treatment of malignant pleural effusion. Semin Oncol 1985;12:54-75.

9 Yasumoto K, Miyazaki K, Nagashima A, et al. Induction of lymphokine-activated killer cells by intrapleural instillation of recombinant interleukin-2 in patients with malignant pleurisy due to lung cancer. Cancer Res 1987;47:2184-7.

10 Sone S, Nakanishi M, Ohmoto Y, et al. Macrophage colonystimulating factor activity in malignant pleural effusions. Chest 1991:99:377-81.

11 Shimokata K, Saka H, Murate T, et al. Cytokine content in pleural effusion. Chest 1991;99:1103-7.

12 Lucivero G, Pierucci G, Bonomo L. Lymphocyte subsets in peripheral blood and pleural fluid. Eur Respir f 1998;1: 337-9.

13 Inoue Y, Shijubo N, Uede T. Induction of killer cells from ymphocytes in pleural effusion of advanced lung cancer patients. Jpn f Cancer Res 1990;81:1012-20.

14 Albera C, Mabritto I, Ghio P, et al. Lymphocytes subpopulation analysis in pleural fluid and peripheral blood in patients with lymphocytic pleural effusions. Respiration 1991;58:65-71.

15 Yanagawa $\mathrm{H}$, Sone S, Nii A, et al. Lymphokine-activated killer induction and its regulation by macrophages in malignant pleural effusions. Fpn f Cancer Res 1989;80: malignant

16 Takahashi K, Sone S, Kimura S, et al. Phenotypes and lymphokine-activated killer activity of pleural cavity lymphocytes of lung cancer patients without malignant effusion. Chest 1993;103:1732-8

17 Takahashi K, Sone S, Saito S, et al. Granulocytemacrophage colony-stimulating factor augments lymphokine-activated killer activity from pleural cavity mononuclear cells of lung cancer patients without malignant effusion. $\mathcal{F p n}$ f Cancer Res 1995;86:861-6.

18 Nakanishi H, Yasumoto K, Nagashima M, et al. Antitumor activity of macrophages in lung cancer patients with special reference to location of macrophages. Cancer Res 1984;44: 5906-9.

19 Kaplan EL, Meier P. Nonparametric estimation from incomplete observations. f Am Statist Assoc 1956;53:45781.

20 Peto R, Pike MC. Conservatism of the approximation sigma (O-E)2-E in the logrank test for survival data or tumor incidence data. Biometrics 1973;29:579-84.

21 Harris EK, Albert A. Survivorship analysis for clinical studies. New York: Marcel Dekker, 1991: 5-125.

22 Chin Y, Janseens J, Vandepitte J, et al. Phenotypic analysis of tumor-infiltrating lymphocytes from human breast cancer. Anticancer Res 1992;12:1463-6.

23 Mulder WM, Stukart MJ, Roos M, et al. Culture of tumourinfiltrating lymphocytes from melanoma and colon carcinoma: removal of tumour cells does not affect tumour specificity. Cancer Immunol Immunother 1995;41:293-301.

24 Schondorf T, Engel H, Lindemann C, et al. Cellular characteristics of peripheral blood lymphocytes and tumorinfiltration lymphocytes in patients with gynecological infiltration lymphocytes in patients with gynecologi

25 Ulrike L, Burger MD, Maximilian P, et al. Recruitment of host CD $8+\mathrm{T}$ cells by tumor-infiltrating lymphocytes and recombinant interleukin-2 during adoptive immunotherapy of cancer. Surgery 1995; 117:325-33.

26 Yoshino I, Yano T, Murata M, et al. Phenotypes of lymphocytes infiltrating non-small cell lung cancer tissues and its variation with histological types of cancer. Lung Cancer 1993;10:13-9.

27 Roussel E, Gingrass MC, Grimm EA, et al. High expression of adhesion molecules/activation markers with little
interleukin-2, interferon gamma, and tumor necrosis factor beta gene activation in fresh tumor-infiltrating lymphocytes from lung adenocarcinoma. Cancer Immunol Immunother 1995;41:1-9.

28 Lacki JK, Markowska J, Wiktorowicz K. Phenotype of lymphocytes in ovarian tumor peritoneal fluid. Eur f Gynecol phocytes in ovarian tum

29 Markowska J, Lacki JK, Jaroszewski J, et al. The usefulness of CD4/CD8 ratio evaluation in monitoring of ovarian of CD4/CD8 ratio evaluation in monitoring of
cancer patients. Eur f Gynecol Oncol 1995;16:54-8.

30 Goedegebuure PS, Eberlein TJ. The role of CD4+ tumor-infiltrating lymphocytes in human solid tumors. Immunol Res 1995;14:119-31.

31 Lee KY, Goedegebuure PS, Linehan DC, et al. Immunoregulatory effects of CD4+ T helper subsets in human melanoma. Surgery 1995;117:365-72. 